Gut and Liver, Vol. 11, No. 5, September 2017, pp. 702-710

\title{
Rifaximin and Propranolol Combination Therapy Is More Effective than Propranolol Monotherapy for the Reduction of Portal Pressure: An Open Randomized Controlled Pilot Study
}

\author{
Yoo Li Lim¹, Moon Young Kim¹, Yoon Ok Jang ${ }^{1,2}$, Soon Koo Baik ${ }^{1,2}$, and Sang Ok Kwon ${ }^{1}$ \\ Departments of ${ }^{1}$ Internal Medicine and ${ }^{2}$ Cell Therapy and Tissue Engineering, Wonju Severance Christian Hospital, Yonsei University Wonju \\ College of Medicine, Wonju, Korea
}

Background/Aims: Non-selective beta blockers (NSBBs) are currently the only accepted regimen for preventing portal hypertension (PHT)-related complications. However, the effect of NSBBs is insufficient in many cases. Bacterial translocation (BT) is one of the aggravating factors of PHT in cirrhosis; therefore, selective intestinal decontamination by rifaximin is a possible therapeutic option for improving PHT. We investigated whether the addition of rifaximin to propranolol therapy can improve hepatic venous pressure gradient (HVPG) response. Methods: Sixty-four cirrhosis patients were randomly assigned to propranolol monotherapy $(n=48)$ versus rifaximin and propranolol combination therapy $(n=16)$. Baseline and post-treatment HVPG values, BT-related markers (lipopolysaccharide [LPS], LPS-binding protein [LBP], interleukin-6 [IL-6], and tumor necrosis factor $\alpha$ [TNF- $\alpha$ ]), serological data, and adverse event data were collected. HVPG response rate was the primary endpoint. Results: Combination therapy was associated with better HVPG response rates than monotherapy ( $56.2 \%$ vs $87.5 \%, p=0.034)$. In combination therapy, posttreatment BT-related markers were significantly decreased (LPS, $p=0.005$; LBP, $p=0.005$; IL-6, $p=0.005$; TNF- $\alpha, p=0.047$ ). Conclusions: Rifaximin combination therapy showed an additive effect in improving PHT compared to propranolol monotherapy. These pilot data suggest that the addition of rifaximin to NSBBs could be a good therapeutic option for overcoming the limited effectiveness of NSBBs. (Gut Liver 2017;11:702-710)

Key Words: Bacterial translocation; Rifaximin; Hypertension, portal; Hepatic venous pressure gradient

\section{INTRODUCTION}

Various clinical manifestations, such as ascites, variceal bleeding, spontaneous bacterial peritonitis, and hepatorenal syndrome develop according to the severity of portal hypertension (PHT) in advanced cirrhosis. ${ }^{1,2}$ Among these complications, variceal bleeding is a potentially fatal complication, and even with appropriate management by endoscope and medical therapy, the mortality is about $15 \%$ to $20 \% .^{3}$ Therefore, control of portal pressure and hemodynamic instability is a crucial component of cirrhosis management. Non-selective beta blockers (NSBB) are currently the only accepted regimen for reducing portal pressure, ${ }^{4-6}$ and the goal of NSBB therapy is to reduce the hepatic venous pressure gradient (HVPG) more than 20\% from the baseline value or to absolute HVPG value under $12 \mathrm{~mm} \mathrm{Hg.}{ }^{7}$ However, many patients do not reach the treatment target, since only $30 \%$ to $50 \%$ of patients achieve a hemodynamic response to NSBB therapy, which is an important limitation of NSBB. ${ }^{5,8,9}$

Bacterial translocation (BT) is defined as the entry of viable bacteria or their products into the regional lymph nodes, the systemic circulation, and possibly extraintestinal organ. ${ }^{10} \mathrm{BT}$ occurs in 25\% to 30\% of cirrhosis patient with hepatic dysfunction, ${ }^{11}$ and BT-related bacterial infections are closely related to PHT and hyperdynamic circulatory syndrome, therefore, control of BT is also important for cirrhosis management.

Intestinal bacterial overgrowth is a main factor promoting BT; selective intestinal decontamination by antibiotics is theoretically relevant for control of BT. Rifaximin is a gastrointestinal selective antibiotic with a broad spectrum of antimicrobial activity and it is not absorbed systemically. ${ }^{12}$ The effectiveness of rifaximin have been proven in management of hepatic en-

Correspondence to: Moon Young Kim

Department of Internal Medicine, Wonju Severance Christian Hospital, Yonsei University Wonju College of Medicine, 20 Ilsan-ro, Wonju 26426, Korea

Tel: +82-33-741-1229, Fax: +82-33-745-1228, E-mail: drkimmy@yonsei.ac.kr

Received on September 27, 2016. Revised on December 27, 2016. Accepted on January 11, 2017. Published online June 27, 2017 pISSN 1976-2283 eISSN 2005-1212 https://doi.org/10.5009/gnl16478

@ This is an Open Access article distributed under the terms of the Creative Commons Attribution Non-Commercial License (http://creativecommons.org/licenses/by-nc/4.0) which permits unrestricted non-commercial use, distribution, and reproduction in any medium, provided the original work is properly cited. 
cephalopathy and spontaneous bacterial peritonitis. ${ }^{13-15}$ These results indicated that application of rifaximin was helpful for modulating BT. However, studies about the effect of rifaximin for improving PHT, which is measured by HVPG, are currently limited, even though this is one of the most reliable surrogate markers of clinical outcomes in cirrhosis patients. ${ }^{16}$ Therefore, in the present study, we investigated additional effects of rifaximin for controlling PHT and the possible biochemical mechanism, using an open-label randomized controlled pilot study.

\section{MATERIALS AND METHODS}

\section{Patients}

Patients between 18 and 70 years of age with advanced cirrhosis who visited the Wonju Severance Christian Hospital, Yonsei University Wonju College of Medicine between January 2011 to July 2013 were considered eligible for the study. The diagnosis of cirrhosis was either liver biopsy proven or clinically suspected based on image studies that included ultrasonography and computerized tomography scans, the presence of varices in the esophagogastroduodenoscopy, and laboratory data. The enrolled patients abstained from alcohol for at least 3 months before enrollment in any cirrhosis etiology. This study included patients who showed HVPG $\geq 12 \mathrm{~mm} \mathrm{Hg}$ among patients who required NSBB prophylaxis to prevent variceal hemorrhage, according to guidelines; ${ }^{17,18}$ patients with small varices with ChildPugh's class $\mathrm{B} / \mathrm{C}$ or presence of red wale marks on varices, patients with medium/large varices or patients that experienced variceal hemorrhaging. Exclusion criteria were as follows: the baseline HVPG value was less than $12 \mathrm{~mm} \mathrm{Hg}$, the presence of spontaneous bacterial peritonitis, refractory ascites, other overt clinical infections, and hepatocellular carcinoma. Patients with a baseline total bilirubin $>5 \mathrm{mg} / \mathrm{dL}$ or creatinine $>2 \mathrm{mg} / \mathrm{mL}$ were excluded. Patients treated with antibiotics or prebiotics during the last 3 months before enrollment in this study, and patients who refused to participate in this study were also excluded. Based on these criteria, 111 patients were considered as study candidates. Among 111 patients who were included in the original trial, 38 patients were excluded, and 73 patients were considered eligible for this study and randomly assigned to receive propranolol monotherapy $(n=54)$ or rifaximin and propranolol combination therapy $(n=19)$ (Fig. 1). BT related markers such as lipopolysaccharide (LPS), and LPS-binding protein (LBP), proinflammatory cytokines such as interleukin-6 (IL-6) and tumor necrosis factor $\alpha(\mathrm{TNF}-\alpha)$ were also measured at baseline in available patients.

\section{Study design}

This study was done prospectively as a randomized openlabeled controlled trial that was conducted at a single center in South Korea. The Institutional Review Board of the Wonju Severance Christian Hospital approved the protocol. Written informed consent to participate in the study was received from all of the participating patients. The study was conducted in accordance with the principles of the Declaration of Helsinki.

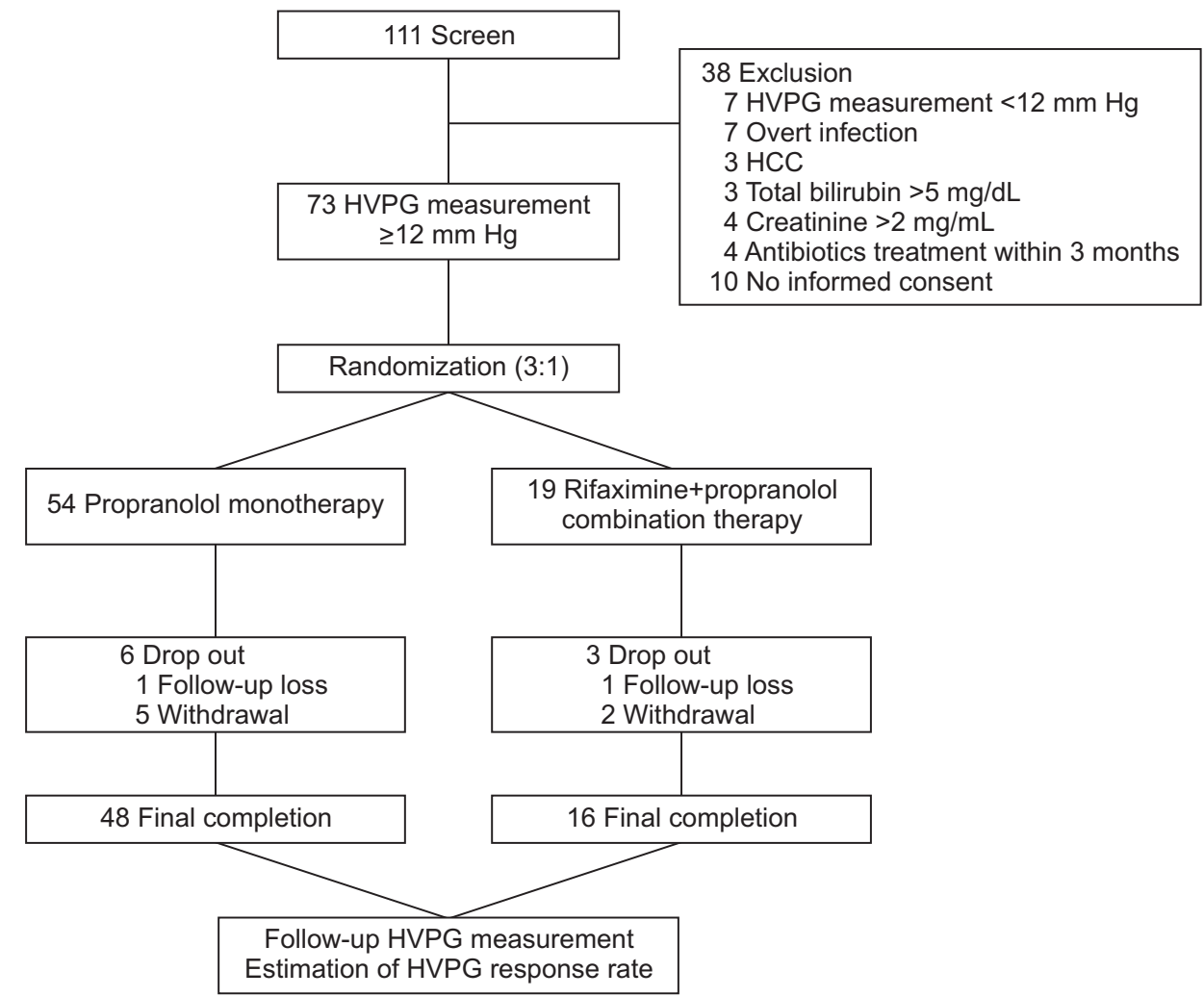

Fig. 1. Flow chart of patient inclusion. HVPG, hepatic venous pressure gradient; HCC, hepatocellular carcinoma. 


\section{1) Randomization and treatment}

Patients were chronologically randomized in a 3:1 fashion using a computer-generated logarithm into either the propranolol monotherapy group (54 patients) or the rifaximin and propranolol combination therapy group (19 patients). The reasons for 3:1 ratio allocation were as follows: First, rifaximin is relatively expensive and its cost was borne by patients because the long-term application of rifaximin was not supported by Korean National Health Insurance Service when this study was performed. Therefore, large population number in rifaximin group was not possible in pilot study. Second, through the previous studies, we could predict the combination group would show better outcome. Finally, previous a few studies have shown that NSBB also could be effective for reducing BT; therefore, we also needed enough numbers of propranolol alone group to confirm the relationship between BT and propranolol monotherapy. The propranolol dosage was started at $40 \mathrm{mg} /$ day and titrated to a maximum of $320 \mathrm{mg} /$ day with a target of 25\% heart rate (HR) reduction or a HR of 55 beats per minute in both groups. In the combination therapy group, additional rifaximin dosing of 1,200 $\mathrm{mg} /$ day was administrated with propranolol. The duration of treatment was 3 months.

\section{2) Primary and secondary end points}

The primary end point was improvement in the HVPG response rate. The HVPG response is defined as the value of HVPG reduced more than 20\% from baseline or to less than 12 $\mathrm{mm} \mathrm{Hg.}{ }^{7}$ The definition of the HVPG response rate is the percent of patients who showed HVPG response in each group. The secondary end point was the change in the HVPG value, HVPG change rate, and change of BT-related markers and proinflammatory cytokines between pre- and posttreatment. Change in the HVPG value is defined as the difference between the HVPG value in pre- and posttreatment. HVPG change rate is defined as the percent of change of HVPG value of the baseline HVPG value.

\section{3) Follow-up}

Patients were followed for 3 months after randomization. During this study, patients did not receive any other medications that could influence the effect of propranolol and rifaximin. Alcohol intake was monitored every week by phone calls with patients and their family, and in their monthly visit to the hospital via liver function tests. Clinical and laboratory test follow-up, and assessment for the presence of side effects were conducted every month. In addition, during each visit, the remaining medications were also counted. During follow-up, six patients in the monotherapy group (follow-up loss, one; withdrawal, five) and three patients in combination therapy group (follow-up loss, one; withdrawal, two) dropped out of the study. After the 3 months, 48 patients in the monotherapy group and 16 patients in the combination therapy group completed treat- ment and entered final analysis. The HVPG, BT-related markers and proinflammatory cytokines were measured again to access the response to treatment (Fig. 1).

\section{HVPG measurement}

After an overnight fast, the right hepatic vein (HV) was catheterized percutaneously through the right femoral vein, and the pressure was recorded in both the free and the wedged position with a 7F balloon-tipped catheter (Arrow International, Erding, Germany). HVPG was determined by subtracting the free HV pressure from the wedged HV pressure. ${ }^{19,20}$ All measurements were performed in at least triplicate, and permanent tracings were obtained on a multichannel recorder. An examiner (Young Joo Kim) with more than 15 years of experience in HVPG measurement performed all of the HVPG procedures and was blinded to clinical information. The coefficient of variation in HVPG measurement at our medical center was 7\%. Clinically significant portal hypertension (CSPH) was defined as HVPG $\geq 10 \mathrm{~mm} \mathrm{Hg}$

\section{BT-related markers and proinflammatory cytokine determination}

To measure BT-related markers and proinflammatory cytokines, peripheral blood samples were collected on the same date of pre- and post-treatment HVPG measurements. Blood samples were separated by centrifugation at $1,500 \times g$ for 15 minutes within 30 minutes of collection. Samples were stored at $-80^{\circ} \mathrm{C}$ until the analysis.

To determine LPS, serum samples were diluted 1:10 with pyrogen-free water and incubated for 10 minutes at $75^{\circ} \mathrm{C}$ to remove serum inhibitors. The concentration of LPS in serum was analyzed using the Limulus Amebocyte Lysate (LAL) assay, a quantitative chromogenic test for detecting endotoxins (QCL-1000 LAL assay; Lonza, Walkersville, MD, USA). The lower detection limit for LPS was $0.1 \mathrm{EU} / \mathrm{mL}$. ELISA assays were used to quantitatively measure the serum concentration of LBP (HK315; Hycult Biotech, Uden, The Netherlands), IL-6 (Human Quantikine kits; R\&D Systems, Minneapolis, MN, USA), and TNF- $\alpha$ (Human Quantikine kits) according to the manufacturers' instructions. The absorbance at the $450-\mathrm{nm}$ light wave was measured in each well with a microplate reader (BioTek ELX; BioTek, Shoreline, WA, USA). The lower limit of detection for LBP was $4.4 \mathrm{ng} / \mathrm{mL}$, IL- 6 was $0.70 \mathrm{pg} / \mathrm{mL}$, and TNF- $\alpha$ was 0.5 $\mathrm{pg} / \mathrm{mL}$. All measurements were performed in duplicate and the mean value is presented

\section{Statistical analysis}

Continuous variables are presented as mean \pm standard deviation. Categorical variables are shown as counts and proportions. Group comparisons in continuous variables were with the Independent t-test and Mann-Whitney $\mathrm{U}$ test, as appropriate. Categorical variables were compared with the chi-square test. 
Repeated measured pre- and post-treatment values were compared with a paired t-test and Wilcoxon signed rank test, as appropriate. A p-value $<0.05$ was considered to be statistically significant. Statistical analysis was conducted using the IBM SPSS version 21.0 (IBM Corp., Armonk, NY, USA).

\section{RESULTS}

The general characteristics are summarized in Table 1 . The general clinical and biological characteristics were well-balanced between groups.

\section{Rifaximin combination therapy results}

The baseline HVPG value was statistically similar in both

Table 1. General Characteristics

\begin{tabular}{|c|c|c|c|}
\hline Characteristic & Monotherapy $(\mathrm{n}=48)$ & Combination therapy $(n=16)$ & p-value \\
\hline Age, yr & $48.79 \pm 9.66$ & $51.19 \pm 9.58$ & 0.393 \\
\hline Male sex & $41(85.4)$ & $15(93.8)$ & 0.667 \\
\hline Etiology & & & 0.701 \\
\hline Alcohol & $28(58.3)$ & $13(81.25)$ & \\
\hline HBV & $10(20.8)$ & $2(12.5)$ & \\
\hline Alcohol+HBV & $8(16.7)$ & $1(6.25)$ & \\
\hline Alcohol+HCV & $1(2.1)$ & 0 & \\
\hline Cryptogenic & $1(2.1)$ & 0 & \\
\hline Child-Pugh score & $7.08 \pm 1.70$ & $6.94 \pm 2.18$ & 0.287 \\
\hline MELD score & $11.44 \pm 4.07$ & $10.13 \pm 3.85$ & 0.262 \\
\hline AST, U/L & $65.69 \pm 35.86$ & $70.64 \pm 43.20$ & 0.693 \\
\hline ALT, U/L & $39.25 \pm 59.92$ & $30.00 \pm 21.41$ & 0.618 \\
\hline Albumin, g/dL & $3.00 \pm 0.35$ & $3.01 \pm 0.43$ & 0.671 \\
\hline Total bilirubin, mg/dL & $2.02 \pm 1.51$ & $3.43 \pm 7.13$ & 0.198 \\
\hline Prothrombin time, INR & $1.33 \pm 0.34$ & $1.22 \pm 0.23$ & 0.206 \\
\hline CRP, mg/dL & $1.01 \pm 1.83$ & $0.74 \pm 0.65$ & 0.719 \\
\hline WBC, $10^{9} / \mathrm{L}$ & $5.47 \pm 2.98$ & $5.27 \pm 1.92$ & 0.805 \\
\hline PMN, $10^{9} / \mathrm{L}$ & $3.53 \pm 2.51$ & $3.11 \pm 1.53$ & 0.529 \\
\hline Platelet, $10^{9} / \mathrm{L}$ & $102.69 \pm 50.25$ & $101.19 \pm 41.70$ & 0.915 \\
\hline Hemoglobin, g/dL & $10.88 \pm 2.05$ & $11.89 \pm 1.99$ & 0.089 \\
\hline Sodium, $\mathrm{mmol} / \mathrm{L}$ & $138.04 \pm 3.13$ & $138.31 \pm 4.03$ & 0.783 \\
\hline Creatinine, $\mathrm{mg} / \mathrm{dL}$ & $0.88 \pm 0.25$ & $0.78 \pm 0.17$ & 0.142 \\
\hline $\mathrm{SBP}, \mathrm{mm} \mathrm{Hg}$ & $116.69 \pm 18.76$ & $117.50 \pm 8.56$ & 0.868 \\
\hline DBP, mm Hg & $73.98 \pm 11.50$ & $71.88 \pm 9.11$ & 0.509 \\
\hline $\mathrm{MBP}, \mathrm{mm} \mathrm{Hg}$ & $88.12 \pm 12.70$ & $87.08 \pm 8.07$ & 0.760 \\
\hline HR, beats/min & $75.02 \pm 11.33$ & $71.31 \pm 9.25$ & 0.241 \\
\hline Dose of propranolol, mg/day & $152 \pm 59.3$ & $127.0 \pm 32.4$ & 0.033 \\
\hline
\end{tabular}

Data are presented as mean \pm SD or number $(\%)$.

HBV, hepatitis B virus; HCV, hepatitis C virus; MELD score, model for end-stage liver disease score; AST, aspartate aminotransferase; ALT, alanine aminotransferase; INR, international normalized ratio; CRP, C-reactive protein; WBC, white blood cell; PMN, polymorphonuclear leukocytes; SBP, systolic blood pressure; DBP, diastolic blood pressure; MBP, mean blood pressure; HR, heart rate.

Table 2. HVPG Value and Response Rates of the Monotherapy and Combination Therapy Groups

\begin{tabular}{lccc}
\hline & Monotherapy $(\mathrm{n}=48)$ & Combination therapy $(\mathrm{n}=16)$ & $\mathrm{p}$-value \\
\hline Baseline HVPG, mm Hg & $17.00 \pm 3.86$ & $17.00 \pm 3.46$ & 1.000 \\
Posttreatment HVPG, mm Hg & $13.52 \pm 4.06$ & $11.19 \pm 4.71$ & 0.060 \\
Change in HVPG, mm Hg & $3.48 \pm 3.85$ & $5.69 \pm 4.19$ & 0.057 \\
HVPG change rate, \% & $18.93 \pm 22.90$ & $34.75 \pm 24.21$ & 0.022 \\
HVPG response rate & $27(56.2)$ & $14(87.5)$ & 0.034 \\
\hline
\end{tabular}

Data are presented as mean \pm SD or number $(\%)$.

HVPG, hepatic venous pressure gradient. 
groups $(\mathrm{p}=1.000)$ (Table 2). After treatment, portal pressure declined significantly in both groups (monotherapy, 17.00 \pm 3.86 to $13.52 \pm 4.06 \mathrm{~mm} \mathrm{Hg}, \mathrm{p}<0.001$; combination therapy, $17.00 \pm 3.46$ to $11.19 \pm 4.71 \mathrm{~mm} \mathrm{Hg} ; \mathrm{p}=0.016$ ) (Fig. 2). After the treatment, the HVPG response rate was significantly greater in the combination therapy group than in the monotherapy group (27/48 patients [56.2\%] vs 14/16 patients [87.5\%], $\mathrm{p}=0.034$ ) (Table 2, Fig. 3A). The change in the HVPG value decreased in the combination therapy group compared with the monotherapy group (3.48 $\pm 3.85 \mathrm{~mm} \mathrm{Hg}$ vs $5.69 \pm 4.19 \mathrm{~mm} \mathrm{Hg}, \mathrm{p}=0.057)$. The HVPG change rate was also significantly greater in the combination therapy group than in the monotherapy group (18.93\% $\pm 22.90 \%$ vs $34.75 \% \pm 24.21 \%, p=0.022$ ) (Fig. 3B).

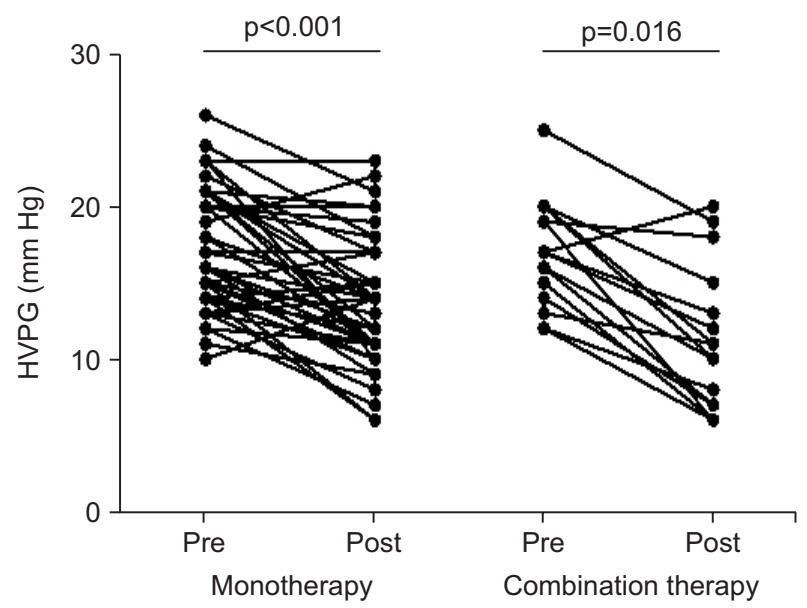

Fig. 2. Pretreatment and posttreatment hepatic venous pressure gradient (HVPG) value comparisons between the propranolol monotherapy group and the combination therapy group (monotherapy group, $17.00 \pm 3.86$ to $13.52 \pm 4.06 \mathrm{~mm} \mathrm{Hg}, \mathrm{p}<0.001$; combination therapy group, $17.00 \pm 3.46$ to $11.19 \pm 4.71 \mathrm{~mm} \mathrm{Hg}, \mathrm{p}=0.016)$.

Pre, pretreatment; Post, posttreatment.

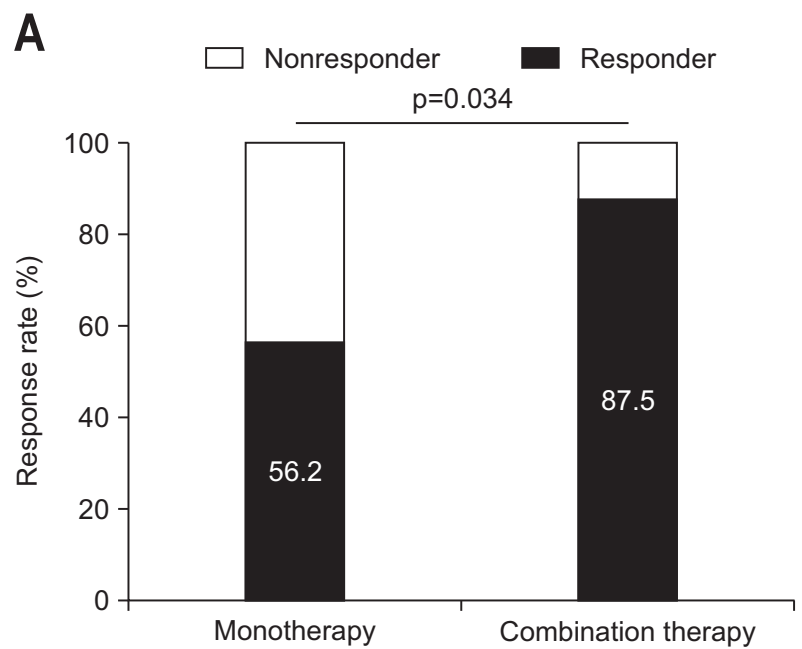

\section{Rifaximin combination therapy for BT-related markers and proinflammatory cytokines}

The BT-related markers and proinflammatory cytokines were measured for 13 patients in the monotherapy group and 10 patients in the combination therapy group. The baseline LPS, LBP, IL-6, and TNF- $\alpha$ were not significantly different between the groups. All BT-related markers and proinflammatory cytokines decreased after treatment regardless of therapy. In the combination therapy group, all BT-related markers and proinflammatory cytokines showed statistically significant decreases after treatment (LPS, $\mathrm{p}=0.005$; LBP, $\mathrm{p}=0.005$; IL-6, p=0.005; TNF- $\alpha$, $\mathrm{p}=0.047$ ) (Table 3). In the monotherapy group, all BT-related markers and proinflammatory cytokines also decreased after treatment (LPS, $\mathrm{p}=0.064$; LBP, $\mathrm{p}=0.055$; IL-6, $\mathrm{p}=0.007$; TNF- $\alpha$, $\mathrm{p}=0.064$ ) (Table 3 ). The change rates of LPS and LBP between the pre- and post-treatment were significantly higher in the combination therapy group than in the monotherapy group (LPS, $\mathrm{p}=0.009$; LBP, $\mathrm{p}=0.002$ ); however, IL-6 and TNF- $\alpha$ results were not statistically significant (Table 3 ).

In subgroup analysis for NSBB responders, LPS, LBP, and IL-6 significantly decreased (LPS, p=0.008; LBP, p=0.008; IL-6, $\mathrm{p}=0.008$ ), and TNF- $\alpha$ also showed a decreasing trend after treatment ( $\mathrm{p}=0.051$ ) in the combination therapy group (Table 4). In the monotherapy group, LPS and IL-6 significantly decreased after treatment (LPS, $p=0.028$; IL-6, $p=0.005$ ), and LBP also had a decreasing trend after treatment $(\mathrm{p}=0.059)$ (Table 4$)$.

\section{Safety}

No significant difference in baseline systolic blood pressure, diastolic blood pressure, and mean blood pressure (MBP) between the monotherapy group and the combination therapy group were observed (Table 1). The reduction in MBP after

B

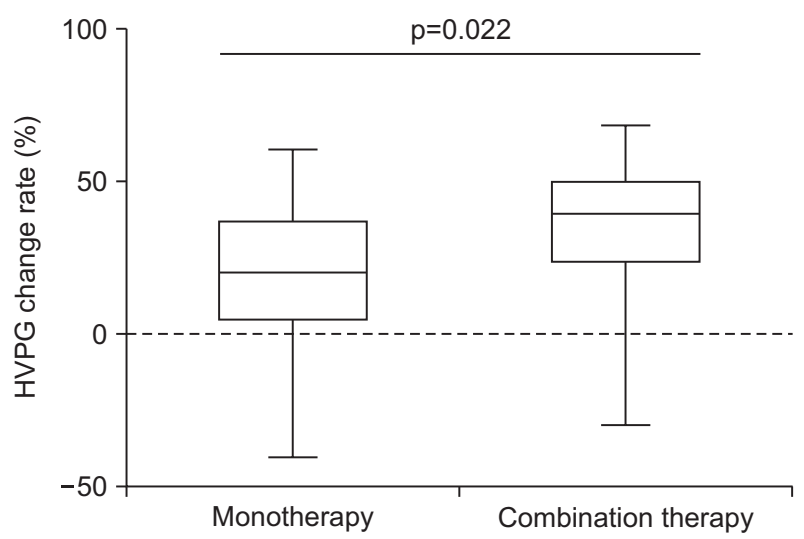

Fig. 3. (A) Comparison of the hepatic venous pressure gradient (HVPG) response rate between the monotherapy and combination therapy groups $(56.2 \%$ vs $87.5 \%, p=0.034)$. (B) HVPG change rate between the monotherapy and combination therapy groups (18.93\% $\pm 22.90 \%$ vs $34.75 \% \pm 24.21 \%, \mathrm{p}=0.022$ ). 
Table 3. BT-Related Markers and Proinflammatory Cytokine Comparison between the Monotherapy and Combination Therapy Groups

\begin{tabular}{|c|c|c|c|c|c|c|c|c|c|}
\hline & \multicolumn{4}{|c|}{ Monotherapy (n=13) } & \multicolumn{4}{|c|}{ Combination therapy $(n=10)$} & \multirow{2}{*}{$p$-value $e^{\ddagger}$} \\
\hline & Pretreatment & Posttreatment & Change rate, $\% *$ & p-value ${ }^{\dagger}$ & Pretreatment & Posttreatment & Change rate, $\% *$ & p-value ${ }^{\dagger}$ & \\
\hline LPS, EU/mL & $1.37 \pm 0.38$ & $1.29 \pm 0.48$ & $5.99 \pm 25.21$ & 0.064 & $1.48 \pm 0.77$ & $0.86 \pm 0.29$ & $34.37 \pm 20.83$ & 0.005 & 0.009 \\
\hline LBP, ng/mL & $20.84 \pm 2.77$ & $19.08 \pm 3.93$ & $8.58 \pm 14.25$ & 0.055 & $21.04 \pm 2.36$ & $15.51 \pm 3.05$ & $26.62 \pm 8.56$ & 0.005 & 0.002 \\
\hline $\mathrm{IL}-6, \mathrm{pg} / \mathrm{mL}$ & $9.65 \pm 3.33$ & $5.52 \pm 4.03$ & $42.85 \pm 27.82$ & 0.007 & $10.31 \pm 4.53$ & $4.78 \pm 2.86$ & $52.76 \pm 22.05$ & 0.005 & 0.366 \\
\hline TNF- $\alpha, p g / m L$ & $4.35 \pm 1.03$ & $3.85 \pm 0.88$ & $9.14 \pm 20.12$ & 0.064 & $4.52 \pm 0.54$ & $3.83 \pm 0.87$ & $14.63 \pm 19.49$ & 0.047 & 0.518 \\
\hline
\end{tabular}

Data are presented as mean \pm SD.

BT, bacterial translocation; LPS, lipopolysaccharide; LBP, LPS-binding protein; IL-6, interleukin-6; TNF- $\alpha$, tumor necrosis factor $\alpha$.

*The rate of change is defined as the percent difference between the pre- and posttreatment values of BT-related markers and proinflammatory cytokines; ${ }^{\dagger} p$-values for the differences between pre- and posttreatment BT-related markers and proinflammatory cytokines; ${ }^{\ddagger} \mathrm{p}$-value of the differences in the rate of change of BT-related markers and proinflammatory cytokines between the monotherapy and combination therapy groups.

Table 4. BT-Related Markers and Proinflammatory Cytokine Comparisons of Hemodynamic Responders in the Monotherapy and Combination Therapy Groups

\begin{tabular}{|c|c|c|c|c|c|c|}
\hline & \multicolumn{3}{|c|}{ Monotherapy $(\mathrm{n}=10)$} & \multicolumn{3}{|c|}{ Combination therapy $(\mathrm{n}=8)$} \\
\hline & Pretreatment & Posttreatment & $\mathrm{p}$-value & Pretreatment & Posttreatment & $\mathrm{p}$-value \\
\hline LPS, EU/mL & $1.26 \pm 0.28$ & $1.09 \pm 0.21$ & 0.028 & $1.45 \pm 0.81$ & $0.78 \pm 0.15$ & 0.008 \\
\hline LBP, ng/mL & $19.96 \pm 2.08$ & $17.82 \pm 3.44$ & 0.059 & $20.52 \pm 1.80$ & $14.94 \pm 2.62$ & 0.008 \\
\hline $\mathrm{IL}-6, \mathrm{pg} / \mathrm{mL}$ & $9.34 \pm 3.44$ & $4.22 \pm 1.55$ & 0.005 & $9.89 \pm 4.58$ & $4.58 \pm 2.95$ & 0.008 \\
\hline $\mathrm{TNF}-\alpha, \mathrm{pg} / \mathrm{mL}$ & $4.36 \pm 1.12$ & $3.80 \pm 0.93$ & 0.139 & $4.51 \pm 0.58$ & $3.73 \pm 0.87$ & 0.051 \\
\hline
\end{tabular}

Data are presented as mean \pm SD.

BT, bacterial translocation; LPS, lipopolysaccharide; LBP, LPS-binding protein; IL-6, interleukin-6; TNF- $\alpha$, tumor necrosis factor $\alpha$.

treatment did not vary between the two groups $(4.43 \pm 12.31 \mathrm{~mm}$ $\mathrm{Hg}$ vs $2.23 \pm 11.52 \mathrm{~mm} \mathrm{Hg}, \mathrm{p}=0.532)$. The baseline HR was similar in both groups $(75.02 \pm 11.33$ beats/min vs $71.31 \pm 9.25$ beats/ min, $p=0.241$ ) (Table 1), and the HR reduction rate was not significantly different in the two groups $(16.19 \pm 11.21$ beats/min vs $15.13 \pm 10.70$ beats/min, $\mathrm{p}=0.634)$. Dizziness and other NSBB related orthostatic hypotension symptoms were observed in eight patients in the monotherapy group and two patients in the combination therapy group. Rifaximin-related severe adverse events were not observed.

\section{DISCUSSION}

In this study, we analyzed the effectiveness of rifaximin and propranolol combination therapy compared with the standard propranolol monotherapy and the impact of this treatment based on hemodynamic (HVPG value) and nonhemodynamic effects (BT-related markers and proinflammatory cytokines).

NSBB is the only generally recommended medication for primary and secondary prevention of variceal bleeding. ${ }^{21}$ Although recently some arguments have been published on the application of NSBB in the advanced cirrhosis, there is still evidence that indicates that NSBB can help prevent other PHT-related complications such as ascites, spontaneous bacterial peritonitis, hepatorenal syndrome, and hepatic encephalopathy., ${ }^{5,16,22}$ However, these positive data were almost all derived from NSBB responders who were identified by estimating hemodynamic response by the HVPG measurement, and the NSBB response rate was only one-third to one-half in these specific cases. ${ }^{5,23} \mathrm{Al}-$ though there have been many trials to improve the low efficacy of NSBB and to identify another more effective agent, no other alternative treatment has been generally accepted, until now.

BT is a critical factor that triggers host immune response and increases serum levels of endotoxin and proinflammatory cytokines that promote hepatic fibrosis and PHT. ${ }^{11,24-26}$ In comparison, PHT promotes BT and endotoxemia due to impaired host immune response, increased bacterial overgrowth and intestinal permeability. ${ }^{25}$ Therefore, PHT and BT have reciprocal interactions and controlling BT has been a focus for controlling PHT. Intestinal decontamination using norfloxacin has shown promising results; ${ }^{27}$ however, the development of quinolone resistant bacteria and extended-spectrum B-lactamase-producing bacteria prevents safe long-term use of norfloxacin. ${ }^{28,29}$

Rifaximin is an antibiotic with broad antimicrobial, high fecal concentration, and low systemic absorption. ${ }^{12,30}$ In cirrhotic patients, the effectiveness of rifaximin has been investigated in many clinical trials that have addressed hepatic encephalopathy remission and prevention. ${ }^{15,31}$ In a previous study, Vlachogiannakos et $a .^{32}$ identified the possibility of rifaximin as a new candidate for controlling PHT based on the results of a single arm study that used rifaximin and HVPG measurement. However, recently, Kimer et al. ${ }^{33}$ reported opposite results with 
similar design through the randomized controlled trial. They showed that rifaximin therapy is not effective to control hemodynamics in the advanced cirrhosis. This is also opposite finding with our present study results. The reasons that rifaximin effect was different can be found in several different points between Vlachogiannakos' or Kimer's study and our present study. In contrast to rifaximin monotherapy in previous studies, we basically applied propranolol to both group and added rifaximin only to combination group in the present study. It is very important point. Even though rifaximin is expected to control BT, we think that rifaximin alone is not enough to reduce HVPG because PHT and sequential hemodynamic changes are not just results of BT but many other more important factors also affect it. Therefore, in this study, we focused on the additional effect of rifaximin in the propranolol-based anti-PHT treatment. In the context, the target population is also different. The present study basically targeted the control of variceal hemorrhage risk based on HVPG values and therefore, this study includes relatively small numbers of ascites patients (11 patients, seven in monotherapy and four in combination group).

In the present study, the rifaximin and propranolol combined therapy led to a significantly higher hemodynamic response rate compared to the propranolol monotherapy group (HVPG response rate, $87.5 \%$ vs $56.2 \%, p=0.034$ ). Even though the propranolol monotherapy response rate in our institution was not bad compared to generally reported data, the difference in treatment groups was apparent. In addition, the change rate of HVPG after treatment between the two groups was also significantly different $(p=0.022)$. These findings suggest that suppression of BT using rifaximin actually resulted in an improved HVPG response rate. The results also indicated that control of BT and the nonhemodynamic effect could be another important target for controlling PHT, and that BT can be controlled which can induce additional effects for controlling PHT in clinical practice.

Improvement in the hemodynamic response using rifaximin was also confirmed by BT-related biomarkers and proinflammatory cytokines (LPS, LBP, IL-6, and TNF- $\alpha$ ). These results were significant even though the analyzed serum samples were relatively small, because this study was pilot design (combination therapy group, $n=10$; monotherapy group, $n=13$ ). We directly analyzed the relationship between hemodynamic responsiveness and BT-related biomarkers and proinflammatory cytokines changes. All of the BT-related markers and proinflammatory cytokines showed statistically significant decreases after rifaximin treatment (Table 3). In the hemodynamic responders in the combined group, LPS, LBP, and IL-6 showed significant decreases after treatment (Table 4); however, in the non-responders there was no significant change. These findings were similar in the propranolol monotherapy group, and the hemodynamic responders also showed significant decreases in the LPS, IL-6 and trends in LBP. These results suggest that the control of BT and the changes of BT-related biomarkers and proinflammatory cytokines can be a critical factor for determining hemodynamic responsiveness, irrespective of treatment group. This also provides a basis for understanding the prognosis and clinical implications of hemodynamic nonresponders and correlations with the BT response. In a previous review, approximately $40 \%$ of hemodynamic nonresponders do not show rebreeding and the BT prevention effect of NSBB has been suspected to be the main factor for this finding and which is going to be important issue for future clinical research to address. ${ }^{34}$

Recently, some investigators showed that NSBB alone can be helpful for preventing BT and BT-related infection, ACLF by improved sympathetic tone, intestinal motility and decreased intestinal permeability. ${ }^{8,35}$ In this study, the propranolol monotherapy group was also associated with a preventive effect of BT and a decrease in BT-related biomarkers and proinflammatory cytokines (Table 3). Although this effect was relatively small compared with the correlations in rifaximin combination therapy, it also showed the possibility that NSBB alone can impact on BT. With the present data, we cannot estimate the proportion of BT and its contribution to PHT, particularly for validating and clarifying the recent "Window Theory of NSBB" that has been identified. ${ }^{16}$ Therefore, additional studies are needed to determine the longitudinal effect of NSBB-induced BT responsiveness.

MBP was mildly reduced after treatment, in both groups, and some patients had orthostatic hypotension symptoms, although no serious adverse events were identified. In previous studies, the adverse events related to receiving rifaximin included nausea, diarrhea, abdominal discomfort, fatigue, and these results were not clinically significant. ${ }^{36,37}$ In our study, serious adverse events related to receiving rifaximin was not identified during the follow-up period.

There are several limitations to this study even though this was performed as a pilot study. First, the allocation of monotherapy and combination therapy group was three to one, so combination group includes relatively small numbers of patients. Second, the small sample size and numbers of serum analysis is also limitation. However, this was a well-designed randomized controlled trial and it can be used as a basis data for a large-scale, double-blind RCT (NCT01897051). Finally, the mean dose of propranolol in monotherapy and combination group were $152 \pm 59.3 \mathrm{mg}$ and $127.0 \pm 32.4 \mathrm{mg}(\mathrm{p}=0.033)$, respectively. The reason why there is statistical difference is not clear and we could not explain logically through this study. This finding is not enough to support that rifaximin also has propranolol dose reduction effect. Some changes of bacterial colonization and composition can be related with the change of pharmacokinetics and effect of propranolol; however, more accurate pharmacological investigation and validation should be preceded.

In conclusion, we analyzed the effectiveness of rifaximin 
and propranolol combination therapy compared to standard propranolol monotherapy on the point of hemodynamic effect (HVPG value) and nonhemodynamic effect (BT-related markers and proinflammatory cytokines). The addition of rifaximin showed an additive effect for reducing HVPG through stronger prevention of BT than standard propranolol monotherapy. Therefore, administration of rifaximin with propranolol in CSPH patients to reduce HVPG can be applied as a therapeutic option for management of portal hypertensive complications and to overcome the limitations of NSBB.

\section{CONFLICTS OF INTEREST}

No potential conflict of interest relevant to this article was reported.

\section{ACKNOWLEDGEMENTS}

This research was supported by a grant from the Korea Health Technology R\&D Project through the Korea Health Industry Development Institute (KHIDI), funded by the Ministry of Health \& Welfare, Republic of Korea (HI15C2364), and also by the Yonsei University Future-Leading Research Initiative of 2014. It was also supported by The Academic Scholarship of the Korean Association for the Study of the Liver.

\section{REFERENCES}

1. La Mura V, Nicolini A, Tosetti G, Primignani M. Cirrhosis and portal hypertension: the importance of risk stratification, the role of hepatic venous pressure gradient measurement. World J Hepatol 2015;7:688-695.

2. Eom YW, Shim KY, Baik SK. Mesenchymal stem cell therapy for liver fibrosis. Korean J Intern Med 2015;30:580-589.

3. Kapoor A, Dharel N, Sanyal AJ. Endoscopic diagnosis and therapy in gastroesophageal variceal bleeding. Gastrointest Endosc Clin N Am 2015;25:491-507.

4. Groszmann RJ, Garcia-Tsao G, Bosch J, et al. Beta-blockers to prevent gastroesophageal varices in patients with cirrhosis. N Engl J Med 2005;353:2254-2261.

5. Tripathi D, Hayes PC. Beta-blockers in portal hypertension: new developments and controversies. Liver Int 2014;34:655-667.

6. Kim G, Eom YW, Baik SK, et al. Therapeutic effects of mesenchymal stem cells for patients with chronic liver diseases: systematic review and meta-analysis. J Korean Med Sci 2015;30:1405-1415.

7. Giannelli V, Lattanzi B, Thalheimer U, Merli M. Beta-blockers in liver cirrhosis. Ann Gastroenterol 2014;27:20-26.

8. Reiberger T, Ferlitsch A, Payer BA, et al. Non-selective betablocker therapy decreases intestinal permeability and serum levels of LBP and IL-6 in patients with cirrhosis. J Hepatol 2013;58:911-921.

9. Kim JH, Kim JM, Cho YZ, et al. Effects of candesartan and propranolol combination therapy versus propranolol monotherapy in reducing portal hypertension. Clin Mol Hepatol 2014;20:376-383.

10. Koutsounas I, Kaltsa G, Siakavellas SI, Bamias G. Markers of bacterial translocation in end-stage liver disease. World J Hepatol 2015;7:2264-2273.

11. Bellot P, Francés R, Such J. Pathological bacterial translocation in cirrhosis: pathophysiology, diagnosis and clinical implications. Liver Int 2013;33:31-39.

12. Koo HL, DuPont HL. Rifaximin: a unique gastrointestinal-selective antibiotic for enteric diseases. Curr Opin Gastroenterol 2010;26:1725.

13. Ponziani FR, Gerardi V, Pecere S, et al. Effect of rifaximin on gut microbiota composition in advanced liver disease and its complications. World J Gastroenterol 2015;21:12322-12333.

14. Bajaj JS, Barrett AC, Bortey E, Paterson C, Forbes WP. Prolonged remission from hepatic encephalopathy with rifaximin: results of a placebo crossover analysis. Aliment Pharmacol Ther 2015;41:3945.

15. Bass NM, Mullen KD, Sanyal A, et al. Rifaximin treatment in hepatic encephalopathy. N Engl J Med 2010;362:1071-1081.

16. La Mura V, Tosetti G, Primignani M, Salerno F. Use of non-selective beta blockers in cirrhosis: the evidence we need before closing (or not) the window. World J Gastroenterol 2015;21:2265-2268.

17. Kim MY, Baik SK, Lee SS. Hemodynamic alterations in cirrhosis and portal hypertension. Korean J Hepatol 2010;16:347-352.

18. Garcia-Tsao G, Bosch J, Groszmann RJ. Portal hypertension and variceal bleeding: unresolved issues. Summary of an American Association for the Study of Liver Diseases and European Association for the Study of the Liver single-topic conference. Hepatology 2008;47:1764-1772.

19. Lebrec D. Methods to evaluate portal hypertension. Gastroenterol Clin North Am 1992;21:41-59.

20. Kim MY, Baik SK, Yea CJ, et al. Hepatic venous pressure gradient can predict the development of hepatocellular carcinoma and hyponatremia in decompensated alcoholic cirrhosis. Eur J Gastroenterol Hepatol 2009;21:1241-1246.

21. Je D, Paik YH, Gwak GY, et al. The comparison of esophageal variceal ligation plus propranolol versus propranolol alone for the primary prophylaxis of esophageal variceal bleeding. Clin Mol Hepatol 2014;20:283-290.

22. Kim G, Cho YZ, Baik SK. Assessment for risk of bias in systematic reviews and meta-analyses in the field of hepatology. Gut Liver 2015;9:701-706.

23. Suk KT. Hepatic venous pressure gradient: clinical use in chronic liver disease. Clin Mol Hepatol 2014;20:6-14.

24. Guarner C, Soriano G. Bacterial translocation and its consequences in patients with cirrhosis. Eur J Gastroenterol Hepatol 2005;17:2731.

25. Fukui H. Gut-liver axis in liver cirrhosis: how to manage leaky gut and endotoxemia. World J Hepatol 2015;7:425-442.

26. Giannelli V, Di Gregorio V, Iebba V, et al. Microbiota and the gutliver axis: bacterial translocation, inflammation and infection in cirrhosis. World J Gastroenterol 2014;20:16795-16810. 
27. Rasaratnam B, Kaye D, Jennings G, Dudley F, Chin-Dusting J. The effect of selective intestinal decontamination on the hyperdynamic circulatory state in cirrhosis: a randomized trial. Ann Intern Med 2003;139:186-193.

28. Fernández J, Acevedo J, Castro M, et al. Prevalence and risk factors of infections by multiresistant bacteria in cirrhosis: a prospective study. Hepatology 2012;55:1551-1561.

29. Tandon P, Delisle A, Topal JE, Garcia-Tsao G. High prevalence of antibiotic-resistant bacterial infections among patients with cirrhosis at a US liver center. Clin Gastroenterol Hepatol 2012;10:12911298.

30. DuPont HL. Introduction: understanding mechanisms of the actions of rifaximin in selected gastrointestinal diseases. Aliment Pharmacol Ther 2016;43 Suppl 1:1-2.

31. Maharshi S, Sharma BC, Srivastava S, Jindal A. Randomised controlled trial of lactulose versus rifaximin for prophylaxis of hepatic encephalopathy in patients with acute variceal bleed. Gut 2015;64: 1341-1342.

32. Vlachogiannakos J, Saveriadis AS, Viazis N, et al. Intestinal decontamination improves liver haemodynamics in patients with alcohol-related decompensated cirrhosis. Aliment Pharmacol Ther 2009;29:992-999.

33. Kimer N, Pedersen JS, Busk TM, et al. Rifaximin has no effect on hemodynamics in decompensated cirrhosis: a randomized, doubleblind, placebo-controlled trial. Hepatology 2017;65:592-603.

34. Thalheimer U, Bosch J, Burroughs AK. How to prevent varices from bleeding: shades of grey--the case for nonselective beta blockers. Gastroenterology 2007;133:2029-2036.

35. Mookerjee RP, Pavesi M, Thomsen KL, et al. Treatment with nonselective beta blockers is associated with reduced severity of systemic inflammation and improved survival of patients with acuteon-chronic liver failure. J Hepatol 2016;64:574-582.

36. Pimentel M, Park S, Mirocha J, Kane SV, Kong Y. The effect of a nonabsorbed oral antibiotic (rifaximin) on the symptoms of the irritable bowel syndrome: a randomized trial. Ann Intern Med 2006;145:557-563.

37. Leevy CB, Phillips JA. Hospitalizations during the use of rifaximin versus lactulose for the treatment of hepatic encephalopathy. Dig Dis Sci 2007;52:737-741. 